\title{
Oxidative Diversification of Steroids by Nature-Inspired Scanning Glycine Mutagenesis of P450BM3 (CYP102A1)
}

\author{
Wenyu Chen, ${ }^{a}$ Matthew J. Fisher, ${ }^{a}$ Aaron Leung, ${ }^{a}$ Yang Cao, ${ }^{a, b}$ and Luet L. Wong $*, a, b$ \\ ${ }^{a}$ Department of Chemistry University of Oxford, Inorganic Chemistry Laboratory, South Parks Road, Oxford OX1 3QR, \\ U.K. \\ ${ }^{b}$ Oxford Suzhou Centre for Advanced Research, Ruo Shui Road, Suzhou Industrial Park, Jiangsu, 215123, P.R. China.
}

\begin{abstract}
Steroidal compounds are some of the most prescribed medicines, being indicated for the treatment of a variety of conditions including inflammation, heart disease and cancer. Synthetic approaches to functionalized steroids are important for generating steroidal agents for drug screening and development. However, chemical activation is challenging because of the predominance of inert, aliphatic $\mathrm{C}-\mathrm{H}$ bonds in steroids. Here, we report the engineering of the stable, highly active bacterial cytochrome $\mathrm{P} 450$ enzyme P450BM3 (CYP102A1) from Bacillus megaterium for the mono- and di-hydroxylation of androstenedione (AD), dehydroepiandrosterone (DHEA) and testosterone (TST). In order to design altered steroid binding orientations, we compared the structure of wild type P450BM3 with the steroid C19-demethylase CYP19A1 with AD bound within its active site and identified regions of the I helix and the $\beta 4$ strand that blocked this binding orientation in P450BM3. Scanning glycine mutagenesis across 11 residues in these two regions led to steroid oxidation products not previously reported for P450BM3. Combining these glycine mutations in a second round of mutagenesis led to a small library of P450BM3 variants capable of selective (up to 97\%) oxidation of AD, DHEA and TST at the widest range of positions $(\mathrm{C} 1, \mathrm{C} 2, \mathrm{C} 6, \mathrm{C} 7, \mathrm{C} 15$ and $\mathrm{C} 16)$ by a bacterial $\mathrm{P} 450$ enzyme. Computational docking of these steroids into molecular dynamics simulated structures of selective P450BM3 variants suggested crucial roles of glycine mutations in enabling different binding orientations from the wild type, including one that closely resembled that of AD in CYP19A1, while other mutations fine-tuned the product selectivity. This approach of designing mutations by taking inspiration from nature can be applied to other substrates and enzymes for the synthesis of natural products and their derivatives.
\end{abstract}

Keywords: steroid oxidation, cytochrome P450, CYP, steroids, protein engineering, enzyme evolution

\section{INTRODUCTION}

Steroids, like nitrogen heterocyclic compounds, are among the most privileged structures in drug discovery. Over 100 steroidal drugs are in clinical use for the treatment of cancer, inflammation and heart disease, amongst other conditions. The biological activity of steroids is differentiated by the oxygenation level of the four rings of the steroidal core and the nature of the side chain at C17. Hydroxylation and general oxy-functionalization of steroids increase their solubility and introduce diversity to their biological effects. ${ }^{1,2}$ Total synthesis of steroids remains impractical. Instead, all steroids are obtained by semisynthetic methods whereby natural steroids are converted to intermediates and end products. ${ }^{3}$ Numerous functional group conversion routes are available for preparing steroid derivatives. ${ }^{4}$ However, these strategies cannot provide general access to highly oxygenated products because of the predominance of $\mathrm{sp}^{3}$ $\mathrm{C}-\mathrm{H}$ bonds in steroids. A notable exception is the application of $\mathrm{C}-\mathrm{H}$ and $\mathrm{C}-\mathrm{C}$ bond oxidation strategies in the synthesis of ouabagenin from cortisone. ${ }^{5,6}$ Baran has reported scalable electrochemical allylic oxidation of steroids and optimization of Schönecker oxidation at the $\mathrm{C} 12$ position of 17 -keto steroids. ${ }^{7,8}$ These approaches have been applied to synthesize polyoxypregnanes and steroid derivatives with expanded rings. ${ }^{8,9}$
Cytochrome P450 (CYP) enzymes catalyze hydroxylation steps in steroid biosynthesis and the formation of myriad oxyfunctionalized steroids in microorganisms that are sources of many steroidal drugs. P450 enzymes utilize two electrons, typically from $\mathrm{NAD}(\mathrm{P}) \mathrm{H}$, to insert one oxygen atom from atmospheric dioxygen into non-activated $\mathrm{sp}^{3} \mathrm{C}-\mathrm{H}$ bonds in a wide range of organic compounds. ${ }^{10}$ Steroid oxidation by microorganisms has been studied extensively. Hydroxylation at most positions is observed in these whole-cell processes but in most cases there is little selectivity and it is not possible to bias the reactions towards desired products. ${ }^{11,12}$ Although recombinant bacterial P450 enzymes offer well-defined systems that are amenable to enzyme evolution to target specific compounds, there are surprisingly few enzymes with steroid oxidation activity. ${ }^{2,13}$ CYP106A2 from Bacillus megaterium catalyzes $15 \beta$ hydroxylation of 3-oxo- $\Delta^{4}$-steroids including androstenedione (AD) and testosterone (TST), ${ }^{14-17}$ and $7 \beta$ hydroxylation of 3hydroxy- $\Delta^{5}$-steroids such as dehydroepiandrosterone (DHEA). ${ }^{18,19}$ CYP260A1 from Sorangium cellulosum So ce56 is a selective $1 \alpha$-hydroxylase of $\mathrm{C}$ - 19 steroids such as $\mathrm{AD}$ and $\mathrm{TST},{ }^{20,21}$ and it has been engineered to give high selectivity for $1 \alpha$ - and $16 \alpha$-hydroxylation of progesterone. ${ }^{22}$ CYP $154 \mathrm{C} 3$ and CYP154C5 selectively oxidize AD, DHEA and TST at the $16 \alpha$ position. ${ }^{23,24}$ CYP102A1 (P450BM3) from B. megaterium has been engineered to oxidize TST to $2 \beta$ - and $15 \beta$-hydroxy-TST, 
and progesterone to $2 \beta$ - and $16 \beta$-hydroxy-progesterone, with $>95 \%$ selectivity. ${ }^{25-30}$ Further evolution led to $>95 \%$ selectivity for $16 \alpha$ and $16 \beta$ oxidation of AD, TST and synthetic steroids. ${ }^{31}$ More recently, a site-saturation mutagenesis study at 15 active site residues led to $>90 \% 7 \beta$-oxidation of TST, AD and related steroids. $^{32}$

CYP102A1 is the most extensively studied P450 enzyme for biotechnological applications due to its single-polypeptide, catalytically self-sufficient nature. ${ }^{33-36}$ It is also readily expressed to high levels in Escherichia coli at cubic-meter scale. ${ }^{37,38}$ CYP102A1 engineering has provided almost total selectivity for $2 \beta-, 7 \beta-, 15 \beta-, 16 \alpha-$, and $16 \beta$-hydroxylation of TST but other oxidation products are formed in trace quantities. ${ }^{28-32}$ Greater diversity of mono- and poly-hydroxylated products will facilitate applications in the synthesis of steroid derivatives. The narrow range of products from previous mutagenesis studies suggested that the engineered active sites allowed a limited number of binding modes for steroids. In order to access different binding orientations, we took advantage of the availability of the crystal structure of AD-bound human CYP19A1 to identify regions and residues in CYP102A1 that blocked this binding mode. Here, we report the use of scanning glycine mutagenesis across 11 residues in these regions to engineer altered steroid binding and increase the product diversity in the oxidation of AD, DHEA and TST by CYP102A1.

\section{RESULTS}

Initial screening of a P450BM3 variant library. AD (1) and DHEA (2) were initially screened for oxidation with a library of 48 CYP102A1 variants. This library was constructed by introducing mutations at two or more active site residues (R47, Y51, S72, V78, F81, A82, F87, A184, I263, A264, E267, A328, A330 and L437, see Supporting Information, Figure S1) to a set of base variants in order to generate enzymes with diverse substrate pocket topologies for screening of unnatural substrates for oxidation. ${ }^{29,39-41}$ Variants were broadly divided into three groups depending on whether they contained mutations $\mathrm{F} 87 \mathrm{~A}$ or $\mathrm{F} 87 \mathrm{~V}$ to create space close to the heme, or the A330P or A330W mutations to restrict this space, or wild typelike. Hydrophobic substitutions were added to these base variants at other residues that were close to the heme (A328, A264) as well as ones further removed (S72, V78, A82, A184, I263, L437), in order to alter the size and shape of the substrate binding pocket. A full list of the variants as well as the screening results are given in Tables S1-S7 in the Supporting Information. Steroid oxidation screening reactions were analyzed by gas chromatography. All products were isolated from preparative scale reactions by flash column chromatography and supercritical fluid chromatography, identified and fully characterized by NMR and HR-MS data (see Supporting Information).

We found that $\mathrm{AD}$ and DHEA oxidation was promoted by the mutations F87A and F87V. ${ }^{29-31}$ Variants A74G/F87V/A184I (GV/A184I), H171L/Q307H/N319Y/F87V (K19/F87V), K19/F87A/A82M, R47L/Y51F/K19/F87A (R19/F87A), and R19/F87A/A184I showed the highest activities (Table 1 \& Supporting Information Table S5). For AD oxidation, the F87V mutation favored the $16 \beta$ alcohol; the most selective variant R47L/Y51F/I401P/F87V (RP/F87V) gave $92 \%$ of $16 \beta$-hydroxy-AD (1f, Table 1, Fig. 1-iii) at $66 \%$ conversion

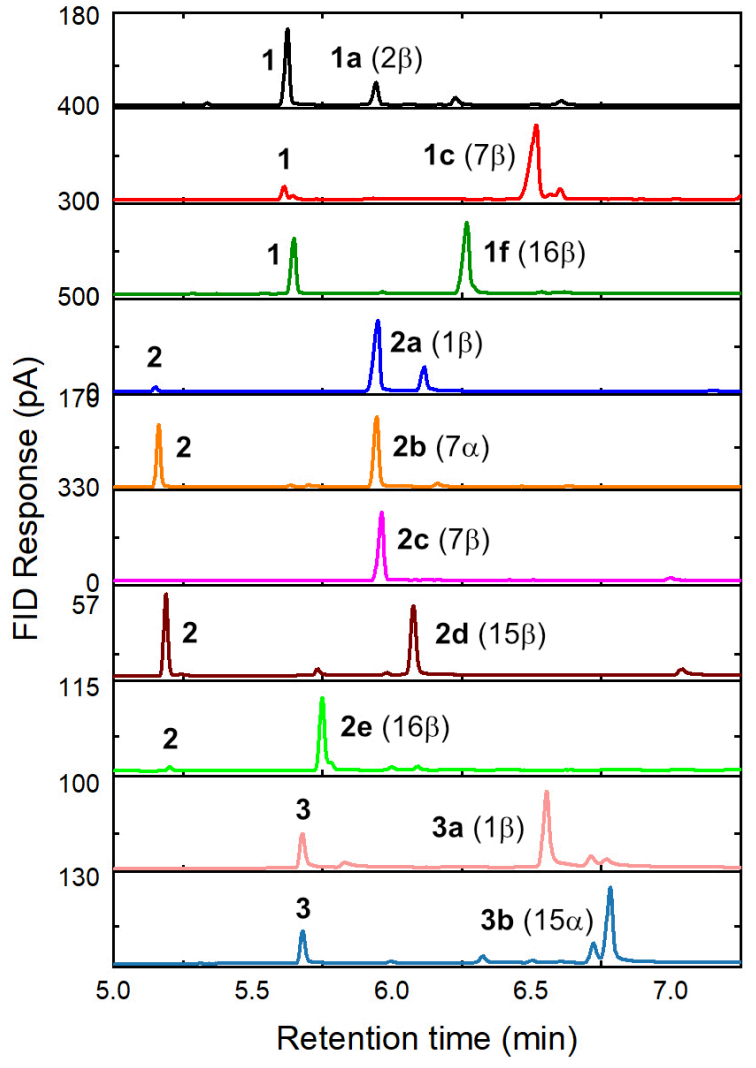

Figure 1. Gas chromatographic analysis of the oxidation of androstenedione $(\mathrm{AD}, \mathbf{1})$, dehydroepiandrosterone (DHEA, 2) and testosterone (TST, 3) catalyzed by CYP102A1 variants: (i) $2 \beta$-hydroxyAD 1a $(60 \%)$ by K19/F87A/A82M/I263G/A264G/A328G; (ii) $7 \beta-$ hydroxy-AD 1c (81\%) by R19/F87A/A184I/T260G/A328G; (iii) $16 \beta$-hydroxy-AD 1f $(92 \%)$ by RP/F87V; (iv) $1 \beta$-hydroxy-DHEA 2a $(23 \%$ ) by R19/F87A/F81W/T260G/A328G; (v) 7 $\alpha$-hydroxyDHEA 2b (93\%) by K19/F87A/A82M/I263G/A328G; (vi) 7ß-hydroxy-DHEA 2c (97\%) by R19/F87A/A184I/T260G/A328G; (vii) 15ß-hydroxy-DHEA 2d (91\%) by R19/F87A/W90V; (viii) $16 \beta$ hydroxy-DHEA 2e (90\%) by GV/A184I; (ix) $1 \beta$-hydroxy-TST 3a (71\%) by R19/F87A/T260G/P329G/A330W; (x) 15 $\alpha$-hydroxyTST 3b (73\%) by GV/A184I/ I263G/A264G/A328G.

(turnover number, TON $=610$ ). The F87A mutation also biased selectivity towards the $16 \beta$ alcohol but it promoted further oxidation. Thus, the R19/F87A variant formed $6 \%$ of $\mathbf{1 f}$ and $81 \%$ of the diol $2 \beta, 16 \beta$-dihydroxy-AD, 1g. On the other hand, the A82M mutation retarded further oxidation, with K19/F87A/A82M giving $77 \%$ of $\mathbf{1 f}$ and $19 \%$ of $\mathbf{1 g}$. Few mutations altered product diversity except for I263G which abolished $16 \beta$ oxidation by F87A-containing variants; the $\mathrm{R} 19 / \mathrm{F} 87 \mathrm{~A} / \mathrm{I} 263 \mathrm{G}$ variant gave $48 \%$ of $6 \beta$-hydroxy-AD, $\mathbf{1 b}$, and $44 \%$ of $16 \alpha$-hydroxy-AD, 1 e.

For DHEA, variants with the F87V mutation also preferred $16 \beta$ oxidation, with GV/A184I giving $90 \%$ of $16 \beta$-hydroxyDHEA, 2e (TON $=760$, Fig. 1-viii, Table 2 \& Supporting Information Table S6). The F87A mutation shifted DHEA hydroxylation away from $16 \beta$ towards the $7 \beta$ - and $15 \beta$-positions. However, the tendency of this mutation to promote 

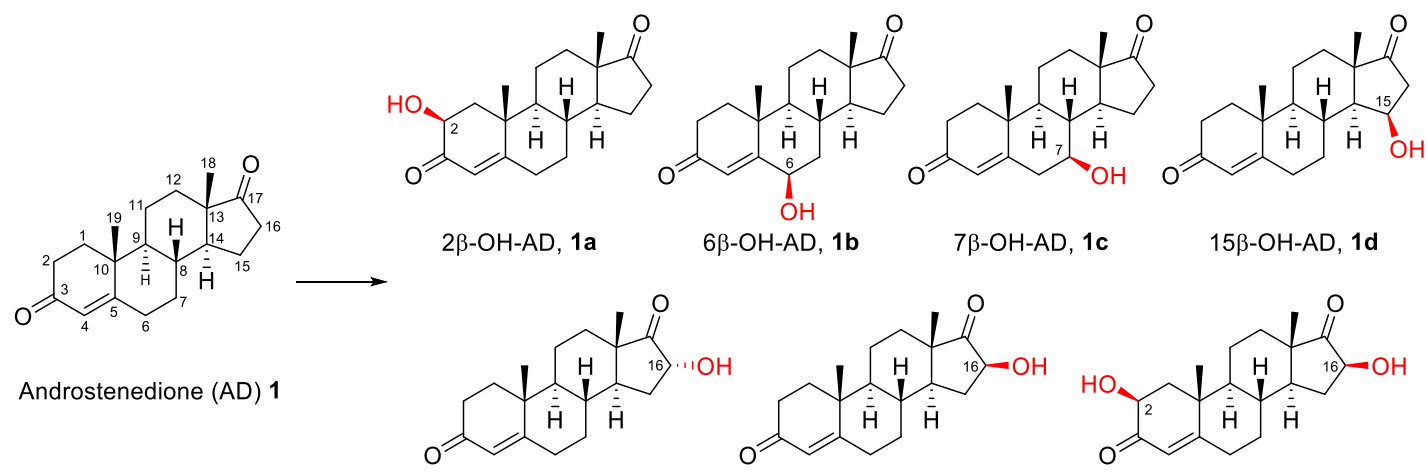

$16 \alpha-\mathrm{OH}-\mathrm{AD}, 1 \mathrm{e}$

$2 \beta, 16 \beta-(\mathrm{OH})_{2}-\mathrm{AD}, \mathbf{1 g}$

\begin{tabular}{|c|c|c|c|c|c|c|c|c|c|c|}
\hline CYP102A1 variant & 1a & 1b & 1c & 1d & 1e & 1f & $1 \mathrm{~g}$ & Others & Conv. & TON \\
\hline R19 F87A A328G & $15 \%$ & - & $3 \%$ & - & - & $20 \%$ & $46 \%$ & $31 \%$ & $54 \%$ & 80 \\
\hline K19 F87A A82M I263G A264G & $59 \%$ & - & - & & - & $25 \%$ & $15 \%$ & $1 \%$ & $36 \%$ & 220 \\
\hline K19 F87A A82M I263G A264G A328G & $60 \%$ & $10 \%$ & $17 \%$ & $13 \%$ & $3 \%$ & - & - & - & $37 \%$ & 230 \\
\hline R19 F87A I263G * & - & $48 \%$ & - & - & $44 \%$ & - & - & $8 \%$ & $35 \%$ & 170 \\
\hline R19 F87A I263G A328G & - & $69 \%$ & - & - & $27 \%$ & - & - & $4 \%$ & $62 \%$ & 430 \\
\hline K19 F87V I263G & - & $78 \%$ & - & - & $22 \%$ & - & - & - & $29 \%$ & 230 \\
\hline R19 F87A A184I T260G & $3 \%$ & - & $12 \%$ & $31 \%$ & - & $20 \%$ & - & $34 \%$ & $69 \%$ & 80 \\
\hline R19 F87A F81W T260G A328G & $5 \%$ & - & $76 \%$ & $8 \%$ & - & $8 \%$ & - & $3 \%$ & $82 \%$ & 630 \\
\hline R19 F87A A184I T260G A328G & - & - & $81 \%$ & - & - & - & - & $19 \%$ & $94 \%$ & 770 \\
\hline R19 F87A A184I L262G & $3 \%$ & - & $2 \%$ & $22 \%$ & - & $14 \%$ & $44 \%$ & $14 \%$ & $98 \%$ & 220 \\
\hline R19 F87A A184I T260G & $3 \%$ & - & $12 \%$ & $31 \%$ & - & $20 \%$ & - & $34 \%$ & $69 \%$ & 210 \\
\hline R19 F87A A264G & $12 \%$ & - & - & $50 \%$ & - & $36 \%$ & - & $2 \%$ & $80 \%$ & 400 \\
\hline R19 F87A I263G * & - & $48 \%$ & - & - & $44 \%$ & - & - & $8 \%$ & $35 \%$ & 160 \\
\hline R19 F87A I263G P329G A330W & - & $43 \%$ & - & - & $48 \%$ & - & - & $9 \%$ & $57 \%$ & 270 \\
\hline K19 F87V* & - & - & - & - & $4 \%$ & $85 \%$ & - & $11 \%$ & $92 \%$ & 790 \\
\hline RP F87V* & - & - & - & - & - & $92 \%$ & - & $8 \%$ & $66 \%$ & 610 \\
\hline R19 F87A* & - & - & - & - & - & $6 \%$ & $81 \%$ & $13 \%$ & $99 \%$ & 800 \\
\hline
\end{tabular}

Screening scale reactions $(0.5 \mathrm{~mL})$ were in $200 \mathrm{mM}$ phosphate buffer (pH 7.9) containing $2 \mu \mathrm{M}$ CYP102 variant, $2 \mathrm{mM}$ AD, GDH (20 $\mathrm{U} / \mathrm{mL})$, glucose $(100 \mathrm{mM})$, and $\mathrm{NADP}^{+}(40 \mu \mathrm{M})$ was added to initiate the reaction in 24-well plates which were shaken at $120 \mathrm{rpm}$ for $16 \mathrm{~h}$ at $20^{\circ} \mathrm{C}$. Conv. is the $\%$ of substrate converted to products. "Others" consists mainly of dihydroxylation products. TON is the turnover number for the product in bold in the enzymatic reactions. * denotes variants in the initial screening library.

further oxidation was retained, e.g. R19/F87A gave $10 \%$ of the $16 \beta$ alcohol $2 \mathrm{e}, 38 \%$ of $15 \beta$-hydroxy-DHEA, $2 \mathrm{~d}$, and $42 \%$ of the diol 7 $\beta, 15 \beta$-dihydroxy-DHEA, 2g. The A264G mutation was notable for favoring $7 \beta$ oxidation, e.g. the K19/F87V/A264G gave $35 \%$ of 7ß-hydroxy-DHEA, 2c, compared to $3 \%$ for $\mathrm{K} 19 / \mathrm{F} 87 \mathrm{~V}$.
Nature-inspired design of scanning glycine mutagenesis. It was clear that variants in the screening library oxidized $\mathrm{AD}$ and DHEA on the lower half of the steroid B and D rings. In an attempt to re-orientate steroid binding and expose the upper part of the $\mathrm{A}$ and $\mathrm{C}$ rings to the oxygen of the CYP102A1 ferryl intermediate, the crystal structure of wild type CYP $102 \mathrm{~A} 1^{42}$ was overlaid with that of CYP19A1 with AD bound within its active site (Figure 2a \& Supporting Information Figure S2). ${ }^{43}$ 
Table 2. Selectivity and turnover number of CYP102A1 variants for the mono-hydroxylation of dehydroepiandrosterone (DHEA, 2).

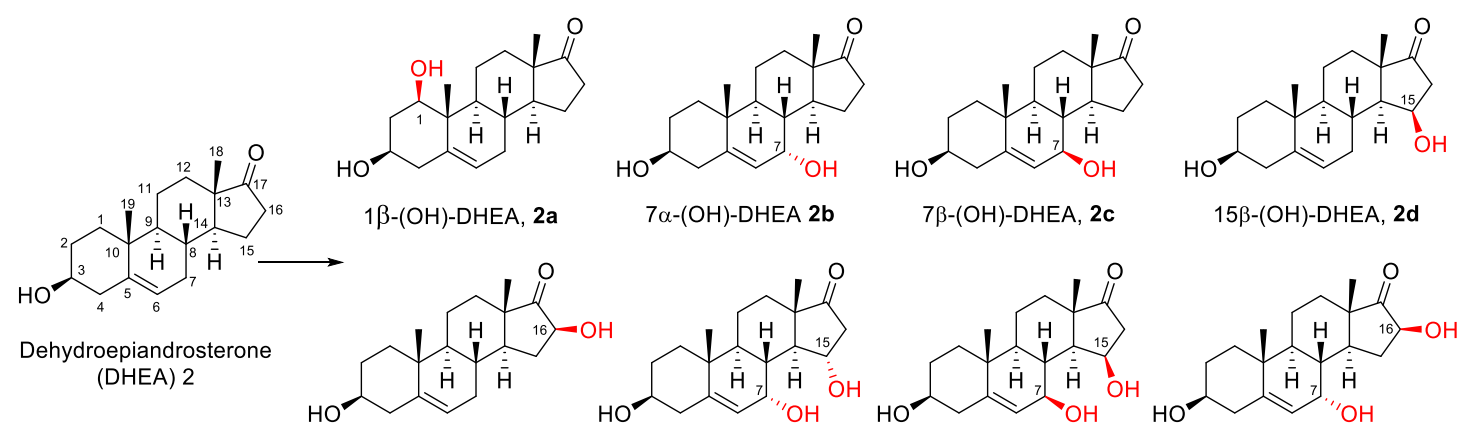

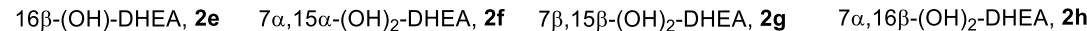

\begin{tabular}{|c|c|c|c|c|c|c|c|c|c|c|}
\hline CYP102A1 variant & $2 a$ & $2 \mathbf{b}$ & $2 c$ & $2 d$ & $2 \mathrm{e}$ & $2 f$ & $2 g$ & Others & Conv. & TON \\
\hline K19 F87A A82M T260G A328G & $18 \%$ & - & $80 \%$ & - & - & - & - & $2 \%$ & $95 \%$ & 170 \\
\hline R19 F87A F81W T260G A328G & $23 \%$ & - & $77 \%$ & & - & - & - & - & $97 \%$ & 220 \\
\hline R19 F87A T260G A328G & $26 \%$ & - & $70 \%$ & - & - & - & - & $4 \%$ & $29 \%$ & 76 \\
\hline R19 F87A T260G A328G P329G A330G & $30 \%$ & $45 \%$ & $22 \%$ & - & - & - & - & $3 \%$ & $37 \%$ & 110 \\
\hline GV A184I I263G A328G & - & $39 \%$ & - & $8 \%$ & - & $40 \%$ & - & $13 \%^{a}$ & $98 \%$ & 390 \\
\hline K19 F87V I263G & - & $58 \%$ & $8 \%$ & $9 \%$ & - & $3 \%$ & $2 \%$ & $20 \%^{b}$ & $68 \%$ & 400 \\
\hline K19 F87A A82M I263G A328G & - & $93 \%$ & - & - & - & - & - & $7 \%$ & $62 \%$ & 580 \\
\hline K19 F87V A264G * & - & - & $35 \%$ & $15 \%$ & $33 \%$ & - & $11 \%$ & $6 \%$ & $87 \%$ & 300 \\
\hline R19 F87A A184I A264G & - & - & $70 \%$ & - & - & - & $27 \%$ & $3 \%$ & $100 \%$ & 700 \\
\hline R19 F87A A184I T260G A328G & - & - & $97 \%$ & - & - & - & - & $3 \%$ & $100 \%$ & 970 \\
\hline R19 F87A * & - & - & - & $37 \%$ & $10 \%$ & - & $42 \%$ & $11 \%$ & $82 \%$ & 310 \\
\hline R19 F87A L262G & - & - & - & $55 \%$ & $33 \%$ & - & $10 \%$ & $2 \%$ & $44 \%$ & 240 \\
\hline R19 F87A T269G & - & - & - & $62 \%$ & $14 \%$ & - & $13 \%$ & $11 \%$ & $44 \%$ & 270 \\
\hline R19 F87A W90V & - & - & - & $91 \%$ & $9 \%$ & - & - & - & $61 \%$ & 560 \\
\hline K19 F87A A82M * & - & - & - & $31 \%$ & $54 \%$ & - & $3 \%$ & $12 \%$ & $92 \%$ & 500 \\
\hline GV A184I * & - & - & - & - & $90 \%$ & - & - & $10 \%$ & $84 \%$ & 760 \\
\hline GV A184I I263G A264G A328G & - & - & - & $12 \%$ & - & $64 \%$ & $5 \%$ & $19 \%$ & $100 \%$ & 640 \\
\hline R19 F87A A184I S270G & - & - & $23 \%$ & - & $3 \%$ & - & $74 \%$ & - & $100 \%$ & 740 \\
\hline
\end{tabular}

Screening scale reactions $(0.5 \mathrm{~mL})$ were in $200 \mathrm{mM}$ phosphate buffer (pH 7.9) containing $2 \mu \mathrm{M}$ CYP102 variant, 2 mM DHEA, GDH (20 $\mathrm{U} / \mathrm{mL})$, glucose $(100 \mathrm{mM})$, and $\mathrm{NADP}^{+}(40 \mu \mathrm{M})$ was added to initiate the reaction in 24-well plates which were shaken at $120 \mathrm{rpm}$ for $16 \mathrm{~h}$ at $20{ }^{\circ} \mathrm{C}$. Conv. is the $\%$ of substrate converted to products. "Others" consists mainly of dihydroxylation products. TON is the turnover number for the product in bold in the enzymatic reactions. ${ }^{*}$ denotes variants in the initial screening library. ${ }^{a}$ contains $5 \%$ of $2 \mathbf{h} .{ }^{b}$ contains $6 \%$ of $\mathbf{2 h}$.

CYP19A1 catalyzes the C19-demethylation of AD to estrone, and of TST to estradiol. AD could not adopt a similar orientation in wild type CYP102A1 because of steric clashes with the side chain of F87, the main chain and side chain of I263 and A264 in the I helix, and the side chain of A328 in the $\beta 4$ strand. This was consistent with the selectivity effect of the mutations F87A, F87V, I263G and A264G in the screening results. The most obvious effect of Gly mutations is to generate space for steroids to adopt different binding orientations. In addition, a Gly residue can cause local distortions and partial unfolding of $\alpha$-helices. Such distortions could increase the flexibility of the substrate pocket for substrate binding. Therefore, although the structural overlay only indicated clashes of AD with I263, A264 and A328, glycine mutations were introduced by site- directed mutagenesis at other residues along the I helix (T260, F261, L262, E267, T269 and S270) 
to both the N- and C-terminus side of I263 and A264, at the B4 strand residues A328, P329 and A330, and combinations thereof. The active variants K19/F87A, K19/F87A/A82M, R19/F87A, R19/F87A/A184I, GV/A184I and K19/F87V were used as templates for this scanning glycine mutagenesis approach.

Second generation variants: oxidative diversification of steroids. The glycine mutations in the new variants (full list in Supporting Information Table S4) did not affect significantly the production level of folded proteins. The new variants showed increased product selectivity and diversity for $\mathrm{AD}$ and DHEA oxidation. The I263G mutation shifted AD oxidation from $16 \beta$ to the $16 \alpha$ and $6 \beta$ positions when combined with F87V, with the K19/F87V/I263G variant forming $78 \%$ of the $6 \beta$-alcohol $\mathbf{1 b}(\mathrm{TON}=230$, Table $1 \&$ Supporting Information Table S5). The A328G and P329G mutations also influenced the $6 \beta / 16 \alpha$ selectivity when combined with F87A, leading to the R19/F87A/I263G/A328G variant which gave $69 \%$ of $\mathbf{1 b}$ at a higher TON (430) than K19/F87V/I263G, while the R19/F87A/I263G/P329G/A330W formed $48 \%$ of the $16 \alpha$ alcohol 1e $(\mathrm{TON}=270)$. The $\mathrm{T} 260 \mathrm{G}$ mutation led to a new product, $7 \beta$-hydroxy-AD, 1c, when combined with $\mathrm{F} 87 \mathrm{~A}$, e.g. $12 \%$ for the R19/F87A/A184I/T260G variant. The A264G and A328G mutations also promoted $7 \beta$ oxidation. Combinations of these increased the selectivity to $81 \%$ of $1 \mathbf{c}(\mathrm{TON}=770)$ for the R19/F87A/A184I/T260G/A328G variant (Fig. 1-ii). The F87A/A264G combination gave two new products - the R19/F87A/A264G formed $50 \%$ of $15 \beta$-hydroxy-AD (1d) and $12 \%$ of the A-ring oxidation product $2 \beta$-hydroxy-AD (1a) at $80 \%$ conversion. The $15 \beta$ alcohol $1 \mathbf{d}$ was also observed when R19/F87A was combined with the I helix mutations T260G (31\%) and L262G (22\%). Since the A328G mutation also led to some $2 \beta$ oxidation (R19/F87A/A328G, $15 \%$ of 1a), combinations of I263G, A264G, A328G, P329G and A330G mutations were added to the template variants. We found that only combinations added to the K19/F87A/A82M template increased $2 \beta$ oxidation selectivity, the highest being $60 \%$ of $1 \mathrm{a}$ $(\mathrm{TON}=230)$ with the K19/F87A/A82M/I263G/A264G/A328G variant (Fig. 1-i).

The $2^{\text {nd }}$ generation variants showed altered selectivity and gave new products with DHEA (Table $2 \&$ Supporting Information Table S6). I helix glycine mutations improved the selectivity for the $15 \beta$ alcohol 2d (R19/F87A/L262G, 55\%; R19/F87A/T269G, 62\%) and for the $7 \beta$ alcohol 2c (R19/F87A/A184I/A264G，70\%; R19/F87A/A184I/T260G, $84 \%$ ). The highest selectivity for $\mathbf{2 d}$ of $91 \%$ (Fig. 1-vii) was found with the R19/F87A/W90V variant identified from screening with the larger library in our collection. The proximity of F87 and W90 might lead to conformational changes that altered DHEA binding. The A328G mutation increased $7 \beta$ selectivity to $97 \%$ of $\mathbf{2 c}(\mathrm{TON}=970)$ with the variant R19/F87A/A184I/T260G/A328G (Fig. 1-vi). By contrast, the I263G mutation favored $7 \alpha$-hydroxy-DHEA, $2 \mathbf{b}$, over the $\beta$ isomer 2c; for example, GV/A184I/I263G showed $42 \%$ C7 oxidation with a $7 \alpha: 7 \beta$ ratio of $84: 16$. The most selective variant $\mathrm{K} 19 / \mathrm{F} 87 \mathrm{~A} / \mathrm{A} 82 \mathrm{M} / \mathrm{I} 263 \mathrm{G} / \mathrm{A} 328 \mathrm{G}$ formed $93 \%$ of $\mathbf{2 b}(\mathrm{TON}=$ 580 ), with no evidence for the $7 \beta$ diastereoisomer (Fig. 1-v). Single glycine mutations did not lead to A-ring oxidation of DHEA. However, the K19/F87A/A82M/T260G/A328G combination variant gave $18 \%$ of $1 \beta$-hydroxy-DHEA, $2 \mathbf{a}(\mathrm{TON}=$ 170). This was increased to $23 \%$ with the variant
R19/F87A/F81W/T260G/A328G at 97\% conversion $(\mathrm{TON}=$ 220 , Fig. 1-iv). The selectivity was slightly higher with other combinations, e.g. R19/F87A/T260G/A328G/P329G/A330G with $30 \%$ but at a lower conversion of $37 \%$.

Dihydroxylation is a common feature of DHEA oxidation by CYP102A1. The diol 7 $\beta, 15 \beta$-dihydroxy-DHEA, 2g, was identified from initial screening. Selectivity for $2 \mathrm{~g}$ was increased to $74 \%$ with the $2^{\text {nd }}$ generation variant $\mathrm{R} 19 / \mathrm{F} 87 \mathrm{~A} / \mathrm{A} 184 \mathrm{I} / \mathrm{S} 270 \mathrm{G}$. The GV/A184I/I263G/A264G/A328G variant gave 64\% (TON $=640$ ) of a new diol $7 \alpha, 15 \alpha$-dihydroxy-DHEA, 2f. On the other hand, the I helix mutations L262G and I263G led to further oxidation at $\mathrm{C} 16$ to form $7 \alpha, 16 \beta$-dihydroxy-DHEA, $\mathbf{2 h}$, with GV/A184I/I263G giving 24\% (Supporting information, TableS6). Interestingly, the F87A/A330W combination also promoted this reaction; with the K19/F87A/A82M/A330W variant forming $33 \% \mathbf{2 h}$.

Formation of $2 \beta$-hydroxy-AD and $1 \beta$-hydroxy-DHEA encouraged us to screen the $2^{\text {nd }}$ generation variants for the oxidation of TST, 3. We found that I helix glycine mutations led to new TST oxidation products for CYP102A1 (Table $3 \&$ Supporting Information Table S7). The GV/A184I/I263G variant gave $23 \%$ of $15 \alpha$-hydroxy-TST, $3 \mathbf{b}$ (Table 3) whereas GV/A184I only formed $15 \beta$-hydroxy-TST (76\%). Inclusion of A264G and the $\beta 4$ strand mutation A328G raised the proportion of $\mathbf{3 b}$ to $73 \%$ for the GV/A184I/I263G/A264G/A328G variant (Fig. 1-x). The T260G mutation promoted TST $1 \beta$-oxidation. The variant R19/F87A/A184I/T260G gave $28 \%$ of $1 \beta$-hydroxyTST (3a) whereas its precursor R19/F87A/A184I formed $56 \%$ of the previously reported $2 \beta$-hydroxy-TST, with no evidence for 3a. Again, addition of $\beta 4$ strand mutations increased the selectivity, to $51 \%$ 3a for R19/F87A/A184I/T260G/A328G and $71 \%(\mathrm{TON}=270)$ for the R19/F87A/T260G/P329G/A330W variant (Fig. 1-ix).

Computational studies. In order to gain insight into CYP102A1-steroid contacts that altered the product profile, molecular dynamics (MD) simulations were performed on variants that oxidized 1-3 with high selectivity. The steroid substrates were docked into the variants using Autodock Vina. The K19/F87A/A82M/I263G/A264G variant was one of the most selective for $2 \beta$ oxidation of $\mathrm{AD}$ ( $59 \%$ of 1a). Docking into six clustered structures across three replicate MD simulations of this variant showed $\mathrm{AD}$ binding poses corresponding to $2 \beta$ oxidation within the top five poses for all clusters. AD adopted similar orientations in all such $2 \beta$ poses (Supporting Information Figure S3). In the lowest energy pose (Figure 2b), C2 of $\mathrm{AD}$ was $3.20 \AA$ from the ferryl oxygen, with $\mathrm{C} 1$ at $3.74 \AA$ and $\mathrm{C} 19$ at $3.29 \AA$. The $\mathrm{C} 3$ carbonyl oxygen contacted the side chains of T260 and the mutated residues M82 and A87, while the $\mathrm{C} 17$ carbonyl oxygen was hydrogen-bonded to the amide $\mathrm{NH}$ of A330. The M82 side chain was in an extended conformation above $\mathrm{A} 87$. Its extensive contacts with $\mathrm{AD}$ were consistent with its importance in promoting $2 \beta$ oxidation. Although $\mathrm{C} 2$ and $\mathrm{C} 19$ were at similar distances from the ferryl oxygen, the more activated $\mathrm{C}-\mathrm{H}$ bond of $\mathrm{C} 2$ was preferentially oxidized. When this docked structure was overlaid with the CYP19A1 structure with bound AD (Figure 2a), we were surprised by how similar this $2 \beta$ pose was to that of AD in CYP19A1. Moreover, the MD simulations suggested that the $\mathrm{I} 263 \mathrm{G}$ and $\mathrm{A} 264 \mathrm{G}$ mutations led to local distortions of 
Table 3. Selectivity and turnover number of CYP102A1 variants for the mono-hydroxylation of testosterone (TST, 3).

\begin{tabular}{|c|c|c|c|c|c|c|c|}
\hline Testosterone (TST) 3 & $1 \beta-(\mathrm{OH})-\mathrm{TST}, \mathbf{3 a}$ & & TST, 3b & & & & \\
\hline CYP102A1 variant & 3a & $\mathbf{3 b}$ & $2 \beta-\mathrm{OH}$ & $15 \beta-\mathrm{OH}$ & Others & Conv. & TON \\
\hline GV A184I * & - & - & $7 \%$ & $76 \%$ & $17 \%$ & $96 \%$ & 960 \\
\hline GV A184I I263G & - & $23 \%$ & - & $37 \%$ & $40 \%$ & $63 \%$ & 150 \\
\hline GV A184I I263G A264G A328G & - & $73 \%$ & - & $14 \%$ & $13 \%$ & $82 \%$ & 600 \\
\hline R19 F87A A184I T260G & $28 \%$ & - & $18 \%$ & $52 \%$ & $2 \%$ & $68 \%$ & 190 \\
\hline R19 F87A A184I T260G A328G & $51 \%$ & $31 \%$ & $4 \%$ & $13 \%$ & $5 \%$ & $100 \%$ & 510 \\
\hline R19 F87A T260G P329G A330W & $71 \%$ & $10 \%$ & - & $11 \%$ & $8 \%$ & $76 \%$ & $270^{a}$ \\
\hline
\end{tabular}

Screening scale reactions $(0.5 \mathrm{~mL})$ were in $200 \mathrm{mM}$ phosphate buffer $(\mathrm{pH} 7.9)$ containing $2 \mu \mathrm{M}$ CYP102 variant, 2 mM TST (TST:enzyme ratio $=1000: 1)$, GDH $(20 \mathrm{U} / \mathrm{mL})$, glucose $(100 \mathrm{mM})$, and $\operatorname{NADP}^{+}(40 \mu \mathrm{M})$ was added to initiate the reaction in 24-well plates which were shaken at $120 \mathrm{rpm}$ for $16 \mathrm{~h}$ at $20^{\circ} \mathrm{C}$. Conv. is the $\%$ of substrate converted to products. ${ }^{*}$ denotes variants in the initial screening library. "Others" consists mainly of dihydroxylation products. TON is the turnover number for the product in bold in the enzymatic reactions except for the first generation variant GV A184I where the TON refers to the formation of all products. ${ }^{a}$ TST:enzyme ratio $=500: 1$.

the helical turn and enabled the I helix in the mutant to move away from the substrate pocket, mimicking the I helix conformation in CYP19A1, thus creating space that allowed AD to bind in a similar position in the CYP102A1 variant to that in CYP19A1.

The R19/F87A/T260G/A328G variant afforded $26 \%$ of $1 \beta$ hydroxy-DHEA (2a) together with $70 \%$ of the $7 \beta$ alcohol $\mathbf{2 c}$. Two of the lowest energy DHEA binding poses corresponded to $1 \beta$ oxidation, while the $3^{\text {rd }}$ lowest energy pose had the $7 \beta$ and $15 \beta \mathrm{C}-\mathrm{H}$ bonds closest to the ferryl oxygen. The allylic nature of $\mathrm{C} 7$ would account for the $7 \beta$ alcohol being the major product. DHEA was bound in similar orientations in the two $1 \beta$ poses. Its $\mathrm{C} 3-\mathrm{OH}$ was within hydrogen-bonding distance of the G260 carbonyl oxygen and the 17-keto group formed a hydrogen bond with the A330 amide NH (Figure 2c). C1 was $3.49 \AA$ from the ferryl oxygen, and $\mathrm{C} 19$ was at $3.26 \AA$ - the more activated $\mathrm{C}-\mathrm{H}$ bond of $\mathrm{C} 1$ was preferentially oxidized. When this binding pose was overlaid with the structure of the R19/F87A precursor (Figure 2d), the C3-OH group of DHEA clashed with the T260 side chain of R19/F87A. Therefore, the I helix glycine mutation T260G was crucial for $1 \beta$ hydroxylation.

R19/F87A/A184I/T260G/A328G was one of the few variants that showed $1 \beta$ oxidation of TST $(51 \%$ of $3 \mathbf{a})$, with the $15 \alpha$ and $15 \beta$ alcohols constituting another $29 \%$ of the products. Docking of TST showed an equal number of poses consistent with $1 \beta$ or $15 \beta$ oxidation within the four lowest energy poses. In the $1 \beta$ poses there was a hydrogen bond between the A330 carbonyl and the C17-OH group of TST while the C 3 carbonyl contacted G260 and the I263 side chain (Figure 2d). C1 and C2 of TST were at similar distances from the ferryl oxygen in all $1 \beta$ poses. In the lowest energy pose, $\mathrm{C} 1$ was $3.65 \AA$ from the ferryl oxygen and $\mathrm{C} 2$ was at $3.51 \AA$. Since $\mathrm{C} 2$ is activated by the 3-keto group, the absence of $\mathrm{C} 2$ oxidation indicated that
TST is bound slightly differently in the variant to the docked pose, with $\mathrm{C} 1$ being closer to the ferryl oxygen.

The $15 \alpha$ alcohol $\mathbf{3 b}$ was a new TST oxidation product for CYP102A1. TST docking poses in the variant GV/A184I/I236G/A264G/A328G (73\% of 3b) were compared to those in $\mathrm{K} 19 / \mathrm{F} 87 \mathrm{~A} / \mathrm{A} 82 \mathrm{M} / \mathrm{I} 263 \mathrm{G} / \mathrm{A} 264 \mathrm{G} / \mathrm{A} 328 \mathrm{G}(56 \%$ of $15 \beta$-hydroxy-TST). As shown in Figure $2 \mathrm{e}$, in the $15 \beta$ poses in the F87A-based variant the $\beta$ side of TST faced the heme, the C17-OH was hydrogen-bonded to the G263 carbonyl, and the A87 methyl group contacted TST carbons. TST was flipped over in the $15 \alpha$ poses in the $\mathrm{F} 87 \mathrm{~V}$-based variant GV/A184I/I263G/A264G/A328G, such that the C18 and C19 methyl groups contacted the V87 side chain to present the $\alpha$ face to the ferryl oxygen (C15 at $3.6 \AA$ ), and the TST C17-OH formed a hydrogen bond with the carbonyl of A330. The $15 \alpha$ pose would not be stable in the F87A-based variant because TST would not contact the A87 side chain methyl (Figure 2f), whereas the $15 \beta$ pose would be destabilized in the F87V-based variant due to steric clashes between TST and V87.

\section{DISCUSSION}

The initial screening library of variants oxidized $\mathrm{AD}$ and DHEA at $\mathrm{C} 6, \mathrm{C} 7, \mathrm{C} 15$ and $\mathrm{C} 16$ on the $\beta$ face (although $\mathrm{AD}$ also formed the $16 \alpha$ alcohol). $\mathrm{C} 15$ and $\mathrm{C} 16$ are the most common positions for steroid oxidation by CYP102A1. Our approach of scanning glycine mutagenesis in the I helix and the $\beta 4$ strand led to new steroid oxidation sites for CYP102A1 ( $2 \beta, 7 \beta$ and $15 \beta$ for AD; $1 \beta, 7 \alpha$ and $15 \alpha$ for DHEA; $1 \beta$ and $15 \alpha$ for TST). Some of the products are accessible with other bacterial P450s. CYP106A2 gives 90\% 7 -oxidation of DHEA ( $v$ s. $97 \%$ by R19/F87A/A184I/T260G/A328G), ${ }^{17,18}$ but no $7 \alpha$ oxidation (vs. 93\% by K19/F87A/A82M/I263G/A328G). CYP154C3 and C5 are selective ( $>95 \%$ ) AD $16 \alpha$ hydroxylases ${ }^{23,24}$ (vs. $48 \%$ by 
a
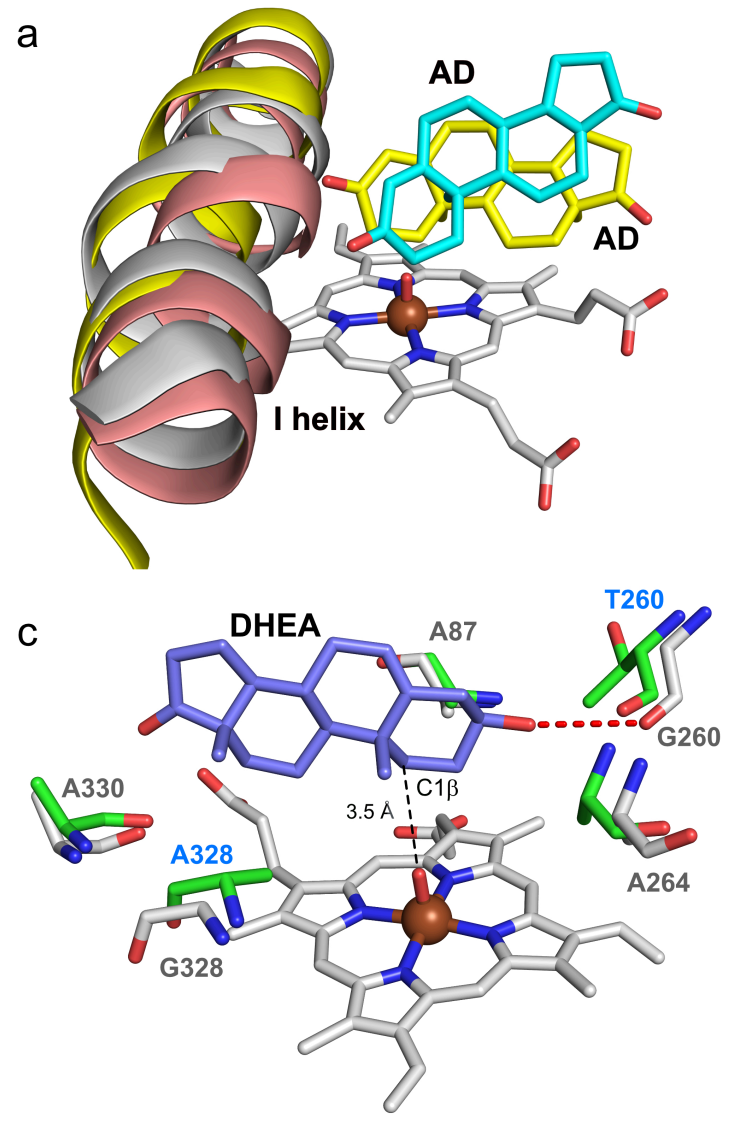

e

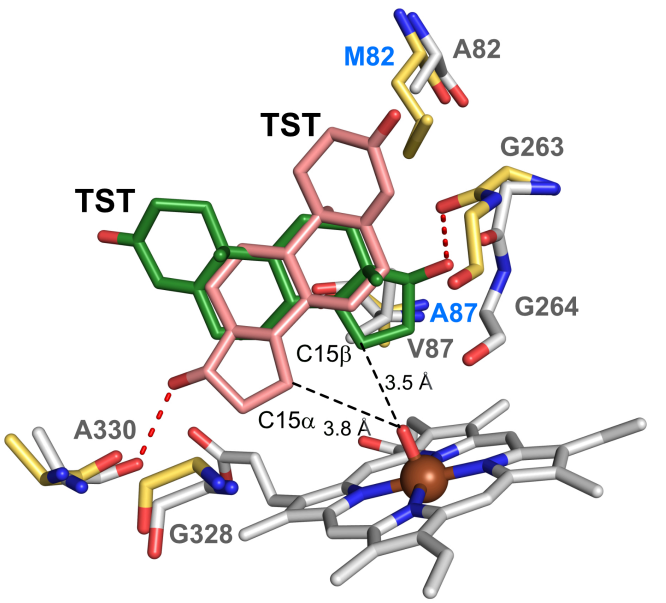

b

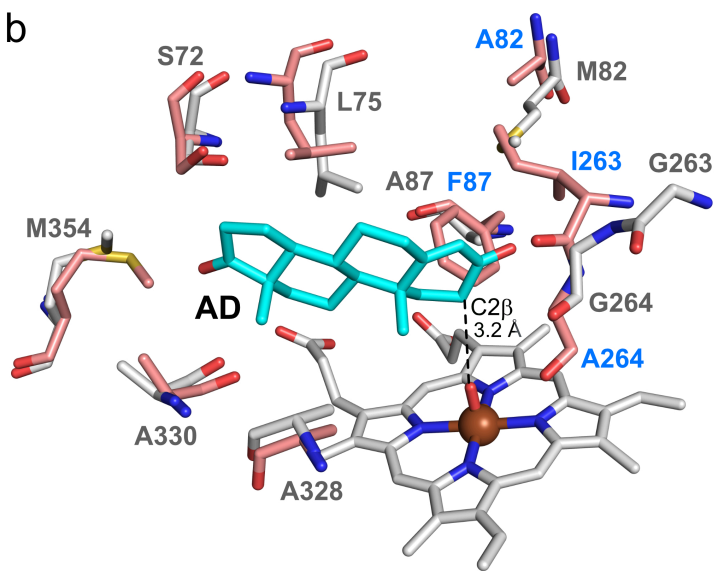

d

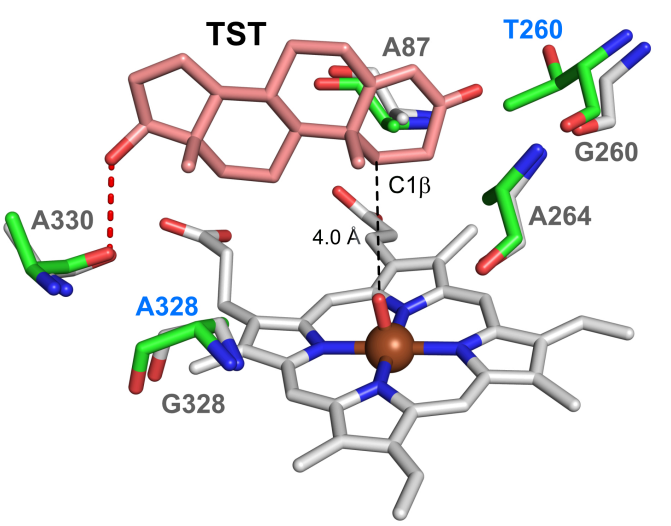

f

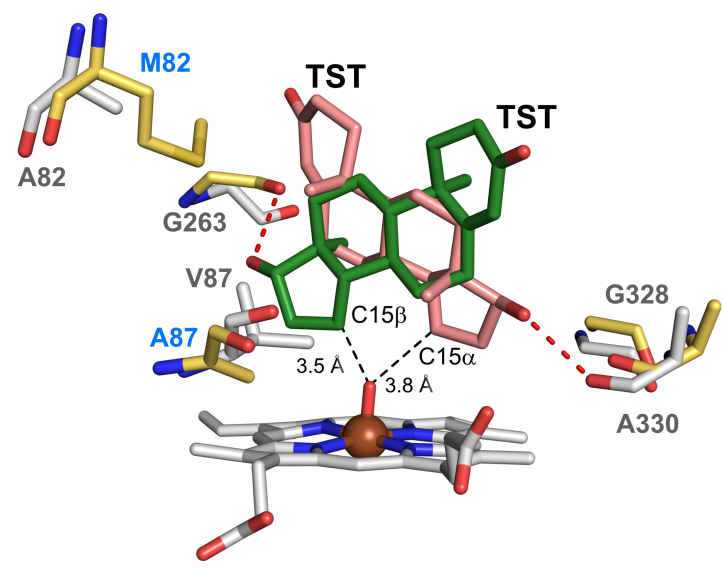

Figure 2. Steroid binding poses from computational docking into MD energy minimized structures of P450BM3 (CYP102A1) variants showing the oxidation site and the distance to the ferryl oxygen. (a) Heme domain of WT P450BM3 ${ }^{42}$ (salmon) overlaid with CYP19A1 ${ }^{43}$ (yellow) bound with $\mathrm{AD}$ (yellow) and with KSK19/A82M/I263G/A264G (grey) with AD (cyan) docked in a $2 \beta$ pose. (b) KSK19/A82M/I263G/A264G (grey) docked with AD (cyan) in a $2 \beta$ pose overlaid with WT P450 $0_{\text {BM3 }}$ (salmon) showing clashes of AD with F87 and I263. (c) R19/F87A/T260G/A328G (grey) docked with DHEA (purple) in a 1 $\beta$ pose showing the H-bond (red dashes) between the $\mathrm{C} 3-\mathrm{OH}$ and the G260 carbonyl. In the R19/F87A precursor variant (green), this C3-OH clashes with T260. (d) R19/F87A/A184I/T260G/A328G (grey) docked with TST (salmon) in a $1 \beta$ pose showing the H-bond between the C17-OH and the A330 carbonyl. The TST C3-carbonyl clashes with T260 in the R19/F87A precursor (green). (e) GV/A184/I263G/A264G/A328G (grey) docked with TST (salmon) in a $15 \alpha$ pose showing the H-bond between the C17-OH and the A330 carbonyl. TST (radium) docked in a $15 \beta$ pose in K19/A82M/F87A/I263G/A264G/A328G (wheat) has a H-bond between the C17-OH and the G263 carbonyl. (f) Back view of (e) showing the flip of TST between the $15 \alpha$ (salmon) and $15 \beta$ (radium) poses. 
R19/F87A/I263G/P329G/A330W); however, similar selectivities have been reported for $16 \alpha$ and $16 \beta$ oxidation of $\mathrm{AD}$ and TST by engineered CYP102A1. ${ }^{30,31} 7 \beta$-Oxidation of AD was only known in fungi ${ }^{44}$ until the recent report by Reetz and co-workers ${ }^{32}$ of $>90 \%$ selectivity by engineered CYP102A1 (vs. $81 \%$ by the R19/F87A/A184I/T260G/A328G variant here). On the other hand, $1 \beta$ and $15 \alpha$ hydroxylation of TST have only been reported in fungi, ${ }^{45}$ likewise for $15 \beta$ oxidation of DHEA, ${ }^{46}$ while there is only one report of $1 \beta$-hydroxy-DHEA by chemical synthesis. ${ }^{47}$ Therefore, the present results, together with previous work, provide the widest range of steroid oxidation products from a bacterial P450 enzyme. Access to multiple monohydroxylated steroids using CYP102A1 is attractive because of the high activity and evolvability of this enzyme. Of the products formed with high selectivity, $6 \beta$-hydroxy-AD is a new hypertensionogenic agent, $7 \alpha$-hydroxy-DHEA potentially promotes weight loss and boosts the activity of immune system, while $7 \beta$-hydroxy-DHEA is a neuroprotective, anti-inflammatory and immune-modulatory agent.

The increase in product selectivity and formation of new products were observed with the $2^{\text {nd }}$ generation library of just 70 variants. Of the I helix glycine mutations, F261G and E267G had little effect. Although this was not surprising since their side chains do not point into the substrate pocket, glycine mutations at other non-active-site residues were effective - L262G and T269G promoted $15 \beta$ oxidation of DHEA while S270G increased substrate conversion. These mutations had to exert their effects by inducing conformational changes in the I helix. The best I helix residues for mutagenesis were the substrate pocket residues T260, I263 and A264. The T260G mutation was particularly important for A-ring oxidation of DHEA and TST. Combination of T260G, I263G, A264G, and I263G/A264G with F87A and F87V increased product diversity. Addition of B4 strand mutations, especially A328G, further expanded the product range. $\mathrm{MD}$ simulations and docking studies indicated that the I helix mutations T260G, I263G and A264G led to local distortion of the I helix to create space for steroid binding in the vicinity of these residues. G260, G263 and A330 were involved in hydrogen bonding with the $\mathrm{C} 3$ and $\mathrm{C} 17$ carbonyls (A330 amide $\mathrm{NH}$ ) and alcohols (G260, G263, and A330 amide CO) at either end of the steroidal framework. Other mutations finetuned steroid binding to alter the product profile. Additional combinations of glycine mutations could provide new product profiles; these are worth pursuing since oxidation at other Aand C-ring positions and the methyl groups, remain to be achieved with CYP102A1. Although further work is required, formation of $7 \alpha, 15 \alpha-, 7 \alpha, 16 \beta$-and $7 \beta, 15 \beta$-dihydroxy-DHEA hint at the possibility of biasing the selectivity for the formation of di-hydroxylated steroids. Polyoxysteroids, such as the pregnane derivative Ouabagenin used in the treatment of congestive heart failure, are challenging to synthesize. ${ }^{5,6,8}$ Poly- and sequential hydroxylation by engineered P450 enzymes may have applications in this area.

In conclusion, scanning and combination glycine mutagenesis of CYP102A1, inspired by the androstenedione binding orientation in the aromatase CYP19A1, has provided access to diverse mono- and di-hydroxylated steroids via direct $\mathrm{C}-\mathrm{H}$ bond oxidation. Difficulties in accessing functionalized steroids by chemical methods is a factor in identifying new biomedical effects from nature-inspired therapeutic compounds. These enzymatic steps complement chemical approaches and could have applications in the synthesis of steroidal agents.

\section{METHODS}

Preparation of enzyme variants. Genes encoding CYP102A1 enzymes were cloned in the pET28+ vector by NcoI and BamHI restriction sites. ${ }^{48}$ Site-directed mutagenesis was carried out by standard PCR-based protocols using a KOD Hot Start DNA Polymerase toolkit from Sigma-Aldrich, UK. The presence of the target mutation(s) was confirmed by DNA sequencing. The relevant plasmid was then transformed into chemically competent $E$. coli BL21 (DE3) for enzyme variant production and subsequent purification as described previously. ${ }^{29,40,41}$

Screening scale reactions. Steroidal substrates were dissolved in an organic solvent (DMSO, EtOH or $\mathrm{MeOH}$ ) and added as a stock, typically at $100 \mathrm{mM}$ concentration. The screening was carried out in $200 \mathrm{mM}$ phosphate buffer ( $\mathrm{pH} 7.9)$ in a volume of $0.5 \mathrm{~mL}$ in 24-well plates. In this reaction system, the final concentration of CYP102A1 variants was $2 \mu \mathrm{M}$ and the substrates was at $2 \mathrm{mM}$. Glucose dehydrogenase (GDH, 20 $\mathrm{U} / \mathrm{mL})$ and glucose $(100 \mathrm{mM})$ were used to regenerate the NADPH cofactor. NADP ${ }^{+}(40 \mu \mathrm{M})$ was added to initiate the reaction. Screening plates were shaken at $120 \mathrm{rpm}$ for $16 \mathrm{~h}$ at $20^{\circ} \mathrm{C}$ and then extracted with $300 \mu \mathrm{L}$ of ethyl acetate. After centrifugation at $14300 \mathrm{~g}$ to separate the phases, the organic extracts were analyzed by GC.

Preparative scale reactions and product characterization. Preparative scale reactions (50-200 mL) with selected enzymesteroid combinations were carried out for 6-16 h under the same conditions as in the initial screening. Progress of the reactions were monitored by GC analysis of organic extracts of 0.5 $\mathrm{mL}$ aliquots. The reaction mixtures were then extracted three times with an equal volume of ethyl acetate, the combined extracts were washed with brine $(20 \mathrm{~mL} \times 3)$, dried with $\mathrm{Na}_{2}\left(\mathrm{SO}_{4}\right)$ and the solvent was removed with a rotary evaporator. The crude mixtures were purified by silica gel column chromatography, eluting with mixtures of DCM and methanol, and by SFC using methanol as the modifier. Analytical thin-layer chromatography was performed on petroleum ether/ethyl acetate solvent system and bands were either visualized under ultraviolet light or with permanganate or phosphomolybdic acid staining reagents. ${ }^{1} \mathrm{H},{ }^{13} \mathrm{C}, \mathrm{COSY}, \mathrm{HSQC}, \mathrm{HMBC}$, NOESY, and 1D nOe NMR spectra were acquired to fully characterize the purified products.

MD simulations and substrate docking. MD simulations were performed on wild type CYP102A1 and variants (GV/A184I, R19/F87A/A264G, R19/F87A/T260G/A328G, R19/F87A/I263G/A328G，GV/A184I/I263G/A264G/A328G, K19/A82M/F87A/I263G/A264G， R19/F87A/A264G/A328G, and R19/F87A/A184I/T260G/A328G) that showed high selectivity for the observed AD, DHEA and TST oxidation products (see ESI). All structures were relaxed via MD simulations performed in GROMACS $2018.6^{49}$ following standard procedures. ${ }^{28,30,31,50}$ Briefly, structures of variants were first prepared by addition of mutations to the WT CYP102A1 crystal structure (PDB code: $1 \mathrm{jpz})^{42}$ via Pymol. $N$-Palmitoylglycine, which was crystallized within the structure, was removed. The protein was prepared using the Amber 99SB*-ILDN force field with TIP3P water. The heme moiety was simulated in the Compound I state, using parameters from Shahrokh, et al. ${ }^{51}$ The protein was placed into the center of an octahedral box, with a minimum distance of $10 \mathrm{~nm}$ to any box edge, solvated with $c a$. 17500 water molecules and the net charge neutralized by addition of $\mathrm{Na}^{+}$ ions. Steepest descent energy minimization was performed until the maximum force was $<500 \mathrm{~kJ} \mathrm{~mol}^{-1} \mathrm{~nm}^{-2}$. Periodic boundary 
conditions were then used to model the system, with electrostatic interactions treated by the particle mesh Ewald method and bond lengths involving $\mathrm{H}$-atoms constrained with the LINCS algorithm. Short-range nonbonded interactions were calculated within a $1 \mathrm{~nm}$ cut-off and a timestep of $2 \mathrm{fs}$ was used. For equilibration steps, all protein backbone $\mathrm{C} \alpha$ atoms were restrained with a positional restraint force constant of $1000 \mathrm{~kJ}$ $\mathrm{mol}^{-1} \mathrm{~nm}^{-2}$. An NVT equilibration step was performed at $298 \mathrm{~K}$ for $100 \mathrm{ps}$, using the modified Berendsen thermostat. This was followed by an NPT equilibration step for 1250 ps utilizing the Berendsen barostat with a time constant of 1 ps. All positional restraints were then removed and $100 \mathrm{~ns}$ of production MD was performed in triplicate using the Parrinello-Rahman barostat with a time constant of $5 \mathrm{ps}$. Structures were recorded every 10 ps. Stability of the simulations was confirmed by monitoring the RMSD of the backbone $\mathrm{C} \alpha$ atoms before the individual trajectories were clustered using the Daura algorithm with a cutoff of $1.2 \mathrm{~nm}$. The three most populated clusters were used as receptor structures for docking studies, which were performed in Autodock Vina. ${ }^{52}$ All water molecules were removed from the structure prior to the docking calculation and both receptor and substrate were treated as rigid entities. The docking site was defined as a $30 \times 30 \times 30 \AA$ box, centered on the ferryl oxygen, and poses were ranked using the Autodock Vina scoring function.

\section{ASSOCIATED CONTENT}

\section{AUTHOR INFORMATION}

\section{Corresponding Author}

*E-mail: luet.wong@chem.ox.ac.uk.

\section{Author Contributions}

W.C., A.L. and Y.C. performed all the molecular biology, microbiology, enzymology, biotransformation and chemical experiments. M.F. performed the in silico experiments to generate energy-minimized structures of the enzyme variants. W.C. performed all the docking experiments. All authors contributed to the writing of the manuscript.

\section{Notes}

The authors declare no competing financial interest.

\section{Supporting Information}

The Supporting Information is available free of charge on the ACS Publications website.

Full detail on experimental procedures, list of enzyme variants and the mutations in each, sequence of oligonucleotides, full results of screening scale reactions in tables, chromatograms of gas chromatography and supercritical fluid chromatography analysis of steroid oxidation reactions, full characterization data for all products, results of molecular dynamics simulations and steroid docking, NMR spectra for all products (PDF)

\section{ACKNOWLEDGMENTS}

The authors acknowledge financial support of this work by the Biotechnology and Biological Sciences Research Council, UK (BB/L024381/1, BB/L013711/1). The authors would like to acknowledge the use of the University of Oxford Advanced Research Computing (ARC) facility in carrying out this work (http://dx.doi.org/10.5281/zenodo.22558).

\section{REFERENCES}

(1) Biellmann, J. F. Enantiomeric Steroids: Synthesis, Physical, And Biological Properties. Chem. Rev. 2003, 103 (5), 2019-2033.

(2) Szaleniec, M.; Wojtkiewicz, A. M.; Bernhardt, R.; Borowski, T.; Donova, M. Bacterial Steroid Hydroxylases: Enzyme Classes, Their Functions and Comparison of Their Catalytic Mechanisms. Appl. Microbiol. Biotechnol. 2018, 102 (19), 8153-8171.

(3) Pines, S. H. The Merck Bile Acid Cortisone Process: The NextTo-Last Word. Org. Proc. Res. Dev. 2004, 8 (5), 708-724.

(4) Hanson, J. R. Steroids: Partial Synthesis in Medicinal Chemistry. Nat. Prod. Rep. 2010, 27 (6), 887-899.

(5) Renata, H.; Zhou, Q.; Baran, P. S. Strategic Redox Relay Enables a Scalable Synthesis of Ouabagenin, a Bioactive Cardenolide. Science 2013, 339 (6115), 59-63.

(6) Renata, H.; Zhou, Q.; Dunstl, G.; Felding, J.; Merchant, R. R.; Yeh, C. H.; Baran, P. S. Development of a Concise Synthesis of Ouabagenin and Hydroxylated Corticosteroid Analogues. J. Am. Chem. Soc. 2015, 137 (3), 1330-1340.

(7) Horn, E. J.; Rosen, B. R.; Chen, Y.; Tang, J.; Chen, K.; Eastgate, M. D.; Baran, P. S. Scalable and Sustainable Electrochemical Allylic C-H Oxidation. Nature 2016, 533 (7601), 77-81.

(8) See, Y. Y.; Herrmann, A. T.; Aihara, Y.; Baran, P. S. Scalable C-H Oxidation with Copper: Synthesis of Polyoxypregnanes. J. Am. Chem. Soc. 2015, 137 (43), 13776-13779.

(9) Zhao, C.; Ye, Z.; Ma, Z. X.; Wildman, S. A.; Blaszczyk, S. A.; Hu, L.; Guizei, I. A.; Tang, W. A General Strategy for Diversifying Complex Natural Products to Polycyclic Scaffolds with Medium-Sized Rings. Nat. Commun. 2019, 10 (1), 4015.

(10) Ortiz de Montellano, P. R., Cytochrome P450: Structure, Mechanism, and Biochemistry 4th ed.; Springer International: Switzerland, 2015.

(11) Donova, M. V.; Egorova, O. V. Microbial Steroid Transformations: Current State and Prospects. Appl. Microbiol. Biotechnol. 2012, 94 (6), 1423-1447.

(12) Niwa, T.; Murayama, N.; Imagawa, Y.; Yamazaki, H. Regioselective Hydroxylation of Steroid Hormones by Human Cytochromes P450. Drug Metab. Rev. 2015, 47 (2), 89-110.

(13) Agematu, H.; Matsumoto, N.; Fujii, Y.; Kabumoto, H.; Doi, S.; Machida, K.; Ishikawa, J.; Arisawa, A. Hydroxylation of Testosterone by Bacterial Cytochromes P450 Using the Escherichia coli Expression System. Biosci. Biotechnol. Biochem. 2006, 70 (1), 307-311.

(14) Berg, A.; Gustafsson, J. A.; Ingelman-Sundberg, M. Characterization of a Cytochrome P-450-Dependent Steroid Hydroxylase System Present in Bacillus megaterium. J. Biol. Chem. 1976, 251 (9), 2831-2838.

(15) Zehentgruber, D.; Hannemann, F.; Bleif, S.; Bernhardt, R.; Lutz, S. Towards Preparative Scale Steroid Hydroxylation with Cytochrome P450 Monooxygenase CYP106A2. Chembiochem 2010, 11 (5), 713-721.

(16) Kiss, F. M.; Schmitz, D.; Zapp, J.; Dier, T. K.; Volmer, D. A.; Bernhardt, R. Comparison of CYP106A1 and CYP106A2 from Bacillus megaterium - Identification of a Novel 11-Oxidase Activity. Appl. Microbiol. Biotechnol. 2015, 99 (20), 8495-8514.

(17) Schmitz, D.; Janocha, S.; Kiss, F. M.; Bernhardt, R. CYP106A2 - A Versatile Biocatalyst with High Potential for Biotechnological Production of Selectively Hydroxylated Steroid and Terpenoid Compounds. Biochim. Biophys. Acta 2018, 1866 (1), 11-22.

(18) Schmitz, D.; Zapp, J.; Bernhardt, R. Steroid Conversion with CYP106A2 - Production of Pharmaceutically Interesting DHEA Metabolites. Microb. Cell. Fact. 2014, 13, 81.

(19) Putkaradze, N.; Kiss, F. M.; Schmitz, D.; Zapp, J.; Hutter, M. C.; Bernhardt, R. Biotransformation of Prednisone and Dexamethasone by Cytochrome P450 Based Systems - Identification of New Potential Drug Candidates. J. Biotechnol. 2017, 242, 101-110. 
(20) Khatri, Y.; Ringle, M.; Lisurek, M.; von Kries, J. P.; Zapp, J.; Bernhardt, R. Substrate Hunting for the Myxobacterial CYP260A1 Revealed New $1 \alpha$-Hydroxylated Products from C-19 Steroids. Chembiochem 2016, 17 (1), 90-101.

(21) Khatri, Y.; Carius, Y.; Ringle, M.; Lancaster, C. R.; Bernhardt, R. Structural Characterization of CYP260A1 from Sorangium cellulosum to Investigate the $1 \alpha$-Hydroxylation of a Mineralocorticoid. FEBS Lett. 2016, 590 (24), 4638-4648.

(22) Khatri, Y.; Jozwik, I. K.; Ringle, M.; Ionescu, I. A.; Litzenburger, M.; Hutter, M. C.; Thunnissen, A. W. H.; Bernhardt, R. Structure-Based Engineering of Steroidogenic CYP260A1 for Stereo- and Regioselective Hydroxylation of Progesterone. ACS Chem. Biol. 2018, 13 (4), 1021-1028.

(23) Bracco, P.; Janssen, D. B.; Schallmey, A. Selective Steroid Oxyfunctionalisation by CYP154C5, a Bacterial Cytochrome P450. Microb. Cell Fact. 2013, 12, 95.

(24) Makino, T.; Katsuyama, Y.; Otomatsu, T.; Misawa, N.; Ohnishi, Y. Regio- and Stereospecific Hydroxylation of Various Steroids at $16 \alpha$ Position of the D Ring by the Streptomyces griseus Cytochrome P450 CYP154C3. Appl. Environ. Microbiol. 2014, 80 (4), 1371-1379.

(25) van Vugt-Lussenburg, B. M.; Damsten, M. C.; Maasdijk, D. M.; Vermeulen, N. P.; Commandeur, J. N. Heterotropic and Homotropic Cooperativity by a Drug-Metabolising Mutant of Cytochrome P450 BM3. Biochem. Biophys. Res. Commun. 2006, 346 (3), 810-818.

(26) van Vugt-Lussenburg, B. M.; Stjernschantz, E.; Lastdrager, J.; Oostenbrink, C.; Vermeulen, N. P.; Commandeur, J. N. Identification of Critical Residues in Novel Drug Metabolizing Mutants of Cytochrome P450 BM3 Using Random Mutagenesis. J. Med. Chem. 2007, 50 (3), 455-461.

(27) Rea, V.; Kolkman, A. J.; Vottero, E.; Stronks, E. J.; Ampt, K. A.; Honing, M.; Vermeulen, N. P.; Wijmenga, S. S.; Commandeur, J. N. Active Site Substitution A82W Improves the Regioselectivity of Steroid Hydroxylation by Cytochrome P450 BM3 Mutants as Rationalized by Spin Relaxation Nuclear Magnetic Resonance Studies. Biochemistry 2012, 51 (3), 750-760.

(28) Venkataraman, H.; Beer, S. B.; Bergen, L. A.; Essen, N.; Geerke, D. P.; Vermeulen, N. P.; Commandeur, J. N. A Single Active Site Mutation Inverts Stereoselectivity of 16-Hydroxylation of Testosterone Catalyzed by Engineered Cytochrome P450 BM3. Chembiochem 2012, 13 (4), 520-523.

(29) Ren, X.; Yorke, J. A.; Taylor, E.; Zhang, T.; Zhou, W.; Wong, L. L. Drug Oxidation by Cytochrome P450BM3: Metabolite Synthesis and Discovering New P450 Reaction Types. Chem. Eur. J. 2015, 21 (42), 15039-15047.

(30) Kille, S.; Zilly, F. E.; Acevedo, J. P.; Reetz, M. T. Regio- and Stereoselectivity of P450-Catalysed Hydroxylation of Steroids Controlled by Laboratory Evolution. Nat. Chem. 2011, 3 (9), 738-743.

(31) Acevedo-Rocha, C. G.; Gamble, C. G.; Lonsdale, R.; Li, A. T.; Nett, N.; Hoebenreich, S.; Lingnau, J. B.; Wirtz, C.; Fares, C.; Hinrichs, H.; Deege, A.; Mulholland, A. J.; Nov, Y.; Leys, D.; McLean, K. J.; Munro, A. W.; Reetz, M. T. P450-Catalyzed Regio- and Diastereoselective Steroid Hydroxylation: Efficient Directed Evolution Enabled by Mutability Landscaping. ACS Catal. 2018, 8 (4), 3395-3410.

(32) Li, A.; Acevedo-Rocha, C. G.; D'Amore, L.; Chen, J.; Peng, Y.; Garcia-Borras, M.; Gao, C.; Zhu, J.; Rickerby, H.; Osuna, S.; Zhou, J.; Reetz, M. T. Regio- and Stereoselective Steroid Hydroxylation at the C7-Position by Cytochrome P450 Monooxygenase Mutants. Angew. Chem. Int. Ed. 2020, DOI:10.1002/anie.202003139.

(33) Jung, S. T.; Lauchli, R.; Arnold, F. H. Cytochrome P450: Taming a Wild Type Enzyme. Curr. Opin. Biotechnol. 2011, 22 (6), 809817 .

(34) Fasan, R. Tuning P450 Enzymes as Oxidation Catalysts. ACS Catal. 2012, 2 (4), 647-666.

(35) Whitehouse, C. J. C.; Bell, S. G.; Wong, L. L. P450BM3 (CYP102A1): Connecting the Dots. Chem. Soc. Rev. 2012, 41 (3), 1218-1260.

(36) Urlacher, V. B.; Girhard, M. Cytochrome P450 Monooxygenases in Biotechnology and Synthetic Biology. Trends Biotechnol. 2019, 37 (8), 882-897.
(37) Pflug, S.; Richter, S. M.; Urlacher, V. B. Development of a FedBatch Process for the Production of the Cytochrome P450 Monooxygenase CYP102A1 from Bacillus megaterium in E. coli. J. Biotechnol. 2007, 129 (3), 481-488.

(38) Kaluzna, I.; Schmitges, T.; Straatman, H.; van Tegelen, D.; Muller, M.; Schurmann, M.; Mink, D. Enabling Selective and Sustainable P450 Oxygenation Technology. Production of 4-Hydroxy-alphaisophorone on Kilogram Scale. Org. Process Res. Dev. 2016, 20 (4), 814-819.

(39) Ren, X. K.; O'Hanlon, J. A.; Morris, M.; Robertson, J.; Wong, L. L. Synthesis of Imidazolidin-4-ones via a Cytochrome P450-Catalyzed Intramolecular C-H Amination. ACS Catal. 2016, 6 (10), 68336837.

(40) Syntrivanis, L. D.; Wong, L. L.; Robertson, J. Hydroxylation of Eleuthoside Synthetic Intermediates by P450BM3 (CYP102A1). Eur. J. Org. Chem. 2018, (45) 6369-6378.

(41) Li, Y.; Wong, L. L. Multi-Functional Oxidase Activity of CYP102A1 (P450BM3) in the Oxidation of Quinolines and Tetrahydroquinolines. Angew. Chem. Int. Ed. 2019, 58 (28), 9551-9555.

(42) Haines, D. C.; Tomchick, D. R.; Machius, M.; Peterson, J. A. Pivotal Role of Water in the Mechanism of P450BM-3. Biochemistry 2001, 40 (45), 13456-13465.

(43) Ghosh, D.; Egbuta, C.; Lo, J. Testosterone Complex and NonSteroidal Ligands of Human Aromatase. J. Steroid Biochem. Mol. Biol. 2018, 181, 11-19.

(44) Yildirim, K.; Kuru, A. Microbial Hydroxylation of Epiandrosterone by Aspergillus candidus. Biocatal. Biotransformation 2017, 35 (2), 120-126.

(45) Peart, P. C.; McCook, K. P.; Russell, F. A.; Reynolds, W. F.; Reese, P. B. Hydroxylation of Steroids by Fusarium oxysporum, Exophiala jeanselmei and Ceratocystis paradoxa. Steroids 2011, 76 (12), 1317-1330.

(46) Koshimura, M.; Utsukihara, T.; Hara, A.; Mizobuchi, S.; Horiuchi, C. A.; Kuniyoshi, M. Hydroxylation of Steroid Compounds by Gelasinospora retispora. J. Mol. Catal. B Enzym. 2010, 67 (1-2), 7277.

(47) Kobayashi, M.; Mitsuhashi, H. Marine Sterols. XIII. Isolation and Synthesis of $1 \beta, 3 \beta, 5,6 \beta$-Tetrahydroxy-5 $\alpha$-androstan-17-one from the Soft Coral Sarcophyton glaucum. Steroids 1982, 40 (6), 673-677.

(48) Whitehouse, C. J. C.; Bell, S. G.; Tufton, H. G.; Kenny, R. J.; Ogilvie, L. C.; Wong, L. L. Evolved CYP102A1 (P450BM3) Variants Oxidise a Range of Non-Natural Substrates and Offer New Selectivity Options. Chem. Commun. 2008, 44 (8), 966-968.

(49) Abraham, M. J.; Murtola, T.; Schulz, R.; Páll, S.; Smith, J. C.; Hess, B.; Lindahl, E. GROMACS: High Performance Molecular Simulations Through Multi-Level Parallelism from Laptops to Supercomputers. SoftwareX 2015, 1-2, 19-25.

(50) Petrovic, D.; Bokel, A.; Allan, M.; Urlacher, V. B.; Strodel, B. Simulation-Guided Design of Cytochrome P450 for Chemo- and Regioselective Macrocyclic Oxidation. J. Chem. Inf. Model. 2018, 58 (4), $848-858$.

(51) Shahrokh, K.; Orendt, A.; Yost, G. S.; Cheatham, T. E., 3rd Quantum Mechanically Derived AMBER-Compatible Heme Parameters for Various States of the Cytochrome P450 Catalytic Cycle. $J$. Comput. Chem. 2012, 33 (2), 119-133.

(52) Trott, O.; Olson, A. J. AutoDock Vina: Improving the Speed and Accuracy of Docking with a New Scoring Function, Efficient Optimization, and Multithreading. J. Comput. Chem. 2010, 31 (2), 455461. 
Table of Contents Graphics

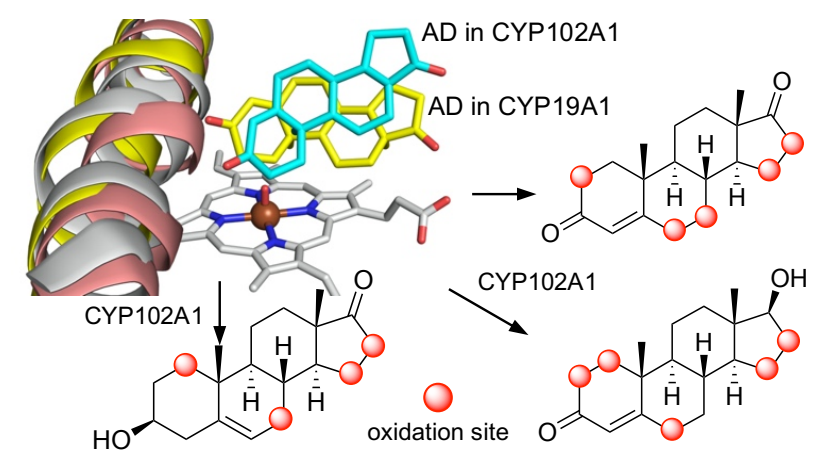

